\title{
ON THE STRUCTURE OF A FINITE SOLVABLE $K$-GROUP
}

\section{MARSHALL KOTZEN}

ABSTRACT. In this note we investigate the structure of a finite solvable $K$-group. It is proved that a finite group $G$ is a solvable $K$-group if and only if $G$ is a subdirect product of a finite collection of solvable $K$-groups $H_{i}$ such that each $H_{i}$ is isomorphic to a subgroup of $G$, and each $H_{i}$ possesses a unique minimal normal subgroup.

This note eliminates several errors in [1]. The statement and the proof of Theorem 1 need correction. The statement of Theorem 1 should read " ... collection of solvable $K$-groups . . " " rather than " . . collection of $K$-groups . . ," while its proof is invalid. In this note we will consider only finite groups with the notation and terminology in [1] assumed to be known.

The following results by $Z$ acher [4] will be useful:

(1) A finite solvable group $G$ is a $K$-group if and only if $G$ contains a series of normal subgroups $1=N_{0}<N_{1}<\cdots<N_{r}=G$ such that each $N_{i+1} / N_{i}$ is a maximal normal nilpotent subgroup of $G / N_{i}$ and $\Phi\left(G / N_{i}\right)=1$ for $i=0,1, \cdots, r-1$.

(2) Each homomorphic image of a solvable $K$-group is a $K$-group.

THEOREM 1. A group $G$ is a solvable $K$-group if and only if $G$ is a subdirect product of a finite collection of solvable $K$-groups $H_{i}$ such that each $H_{i}$ is isomorphic to a subgroup of $G$, and each $H_{i}$ possesses a unique minimal normal subgroup.

Proof. Considering a solvable group $G$, we know that $G$ is a subdirect product of a finite collection of solvable groups $H_{i}$ such that for all $i, H_{i}$ has a unique minimal normal subgroup. Adding the condition that $G$ is a $K$-group, we denote by $A$ the kernel of the projection of $G$ onto $H_{i}$ for some $i$. In particular, using the normality of $A$ in the $K$ group $G$, it must follow that there exists a subgroup $B$ of $G$ such that $G=A B$ with $A \cap B=1$. In particular, we observe that $B \cong H_{i}$, i.e. the

Received by the editors November 18, 1969.

AMS 1969 subject classifications. Primary 2000; Secondary 2025, 2027, 2052.

Key words and phrases. Solvable group, $K$-group, subdirect product, unique minimal normal subgroup, series, maximal normal nilpotent subgroup, Frattini subgroup, Fitting group, elementary abelian, $G$ splits over $F(G)$, completely reducible, direct product. 
direct factors in the subdirect product are isomorphic to subgroups of $G$. Applying (2) we note that $H_{i}$ is a $K$-group. Thus we have established the necessity of the conditions of the theorem.

The sufficiency of the conditions of the above theorem follows by induction on the order of $G$. Suppose the result holds for all groups of order less than $G$. We denote the projection of $G$ on $H_{i}$ by $\pi_{i}$ and the kernel of $\pi_{i}$ by $A_{i}$, and note that $\bigcap_{i \in I} A_{i}=1$. Since $H_{i}$ is a $K$-group, $\Phi\left(H_{i}\right)=1$, and since $\Phi(G) \pi_{i} \leqq \Phi\left(H_{i}\right)$ for all $i \in I$, then $\Phi(G) \leqq \bigcap_{i \in I} A_{i}$ $=1$. Thus for each subgroup $N$ normal in $G, \Phi(N)=1$ (see [2]). But this implies that the Fitting group $F(G)$ is elementary abelian, and that $G$ splits over $F(G)$. That is, there exists a subgroup $C$ such that $G=F(G) \cdot C$ with $F(G) \cap C=1$. Note that $G$ is clearly solvable, and we are assuming $G \neq 1$; thus $\Phi(G)=1<F(G)$. Thus the order of $C$ is less than the order of $G$. Since each $H_{i}$ is a solvable $K$-group, $F\left(H_{i}\right)$ is completely reducible. Thus $F\left(H_{i}\right)$ must be the unique minimal normal subgroup of $H_{i}$ for each $i$. Now we note that $H_{i}=\left(F(G) \pi_{i}\right)\left(C \pi_{i}\right)$. If $F(G) \pi_{i} \cap C \pi_{i}=1$, then $H_{i} / F(G) \pi_{i} \cong C \pi_{i}$. Thus $C \pi_{i}$ is a solvable $K$ group. If $F(G) \pi_{i} \cap C \pi_{i}=B_{i} \neq 1$, then $B_{i} \leqq F(G) \pi_{i} \leqq F\left(H_{i}\right)$, and $B_{i} \neq 1$ implies $B_{i}=F\left(H_{i}\right)$. Furthermore since $F(G) \pi_{i} \leqq F\left(H_{i}\right)$, it follows that $F(G) \pi_{i} \leqq C \pi_{i}=H_{i}$. (I.e. since $F(G) \pi_{i} \leqq F\left(H_{i}\right)=B_{i}$, and $B_{i} \leqq C \pi_{i}$; $F(G) \pi_{i} \leqq C \pi_{i}$. Furthermore since $H_{i}=\left(F(G) \pi_{i}\right)\left(C \pi_{i}\right), g \in H_{i}$ implies $g=f k$ where $f \in F(G) \pi_{i}, k \in C \pi_{i}$. Thus $f \in F\left(H_{i}\right), k \in C \pi_{i}$ implies $f \in B_{i} \leqq C \pi_{i}$, and $k \in C \pi_{i}$ and all this implies $f k=g \in C \pi_{i}$.) Again $C \pi_{i}$ is a solvable $K$-group. Since for each $i \in I, C \pi_{i}$ is a solvable $K$-group, it follows that each $C \pi_{i}$ is a subdirect product of solvable $K$-groups each having precisely one minimal normal subgroup. If we combine the $H_{i}$ for which $C \pi_{i}=H_{i}$ together with the direct factors in the subdirect product associated with those $C \pi_{i}$ for which $C \pi_{i} \neq H_{i}$, a direct product can be formed for which $C$ is a subdirect product of solvable $K$-groups containing precisely one minimal normal subgroup where we use composition of mappings where necessary. By the inductive hypothesis $C$ is a solvable $K$-group. But this implies that in $G$ $=F(G) \cdot C$ there exists a series of subgroups $1=N_{0}<N_{1}<\cdots<N_{r}$ $=G$ such that $N_{i} / N_{i-1}$ is the maximal normal nilpotent subgroup of $G / N_{i-1}$ and $\Phi\left(G / N_{i-1}\right)=1$. Now using Zacher's result (1) above, we note that $G$ is a $K$-group.

I thank Professor Bechtell for suggesting this paper to me.

\section{BiBLIOGRAPHY}

1. H. Bechtell, $A$ note on finite solvable $K$-groups, Proc. Amer. Math. Soc. 17 (1966), 1447-1450. MR 35 \#4302. 
2. W. Gaschütz, Über die $\Phi$-Untergruppe endlichen Gruppen, Math. Z. 58 (1953), 160-170. MR 15, 285.

3. M. Suzuki, Structure of a group and the structure of its lattice of subgroups, Ergebnisse der Math., Heft 10, Springer-Verlag, Berlin, 1956. MR 18, 715.

4. G. Zacher, Caratterizzazione dei gruppi risolubili d'ordine finite complementati, Rend. Sem. Mat. Univ. Padova 22 (1953), 113-122. MR 15, 286; MR 16, 1336.

State College at Worcester, Worcester, Massachusetts 01602 